This document is confidential and is proprietary to the American Chemical Society and its authors. Do not copy or disclose without written permission. If you have received this item in error, notify the sender and delete all copies.

\title{
A mid-infrared spectroscopy platform based on GaAs/AlGaAs thin-film waveguides and quantum cascade lasers
}

\begin{tabular}{|r|l|}
\hline Journal: & Analytical Chemistry \\
\hline Manuscript ID & ac-2015-04144b.R1 \\
\hline Manuscript Type: & Technical Note \\
\hline Date Submitted by the Author: & n/a \\
\hline Complete List of Authors: & $\begin{array}{l}\text { Sieger, Markus; University of Ulm, Institute of Analytical and Bioanalytical } \\
\text { Chemistry } \\
\text { Haas, Julian; Ulm University, Institute of Analytical and Bioanalytical } \\
\text { Chemistry } \\
\text { Jetter, Michael; University of Stuttgart, IHFG } \\
\text { Michler, Peter ; Univ Stuttgart } \\
\text { Godejohann, Matthias; MG Optical Solutions, } \\
\text { Mizaikoff, Boris; Ulm University, Institute of Analytical and Bioanalytical } \\
\text { Chemistry }\end{array}$ \\
\hline
\end{tabular}




\section{Introduction}

Mid-infrared (MIR, 2.5-20 $\mu \mathrm{m}$ ) spectroscopy is nowadays considered a routine analysis and sensing tool for chemical detection and identification in gases, liquids, and solids. ${ }^{1,2}$ Besides transmission-absorption spectroscopy, which is frequently limited by strong background matrix absorptions, infrared attenuated total reflection spectroscopy (IR-ATR) has emerged as a viable and frequently used measurement strategy. ${ }^{3}$ IR-ATR is based on the principle of total internal reflection with analyte molecules probed by the evanescent field, which exponentially decays from the waveguide surface into the probed adjacent medium. ${ }^{4}$ During the last decades, progress in MIR spectroscopy has focused in part on system miniaturization, and has led to a significant reduction in the dimensions of both, analytical device and sample volume. ${ }^{5}$ However, appropriate strategies for the miniaturization of each optical component of the sensing system are required for facilitating future on-chip MIR sensor technology. Compact midinfrared spectroscopic and sensing systems utilizing IR-ATR principles generally require three main components: (i) a MIR light source, (ii) waveguide structures that propagate radiation and frequently also serve as active signal transducers, and (iii) a MIR detector. Among the available MIR radiation sources, the quantum cascade laser (QCL) is nowadays accepted as the most advanced light source technology for compact MIR sensing applications. ${ }^{6,7}$ QCLs are semiconductor lasers based on quantum heterostructures generating light emission via intersubband transitions. QCLs provide compact dimensions, high output power, long lifetime and stability, and availability across almost the entire MIR spectral regime. In addition, coupling to an external cavity provides exceedingly generous wavelength tunability up to $>250 \mathrm{~cm}^{-1}$ for a single laser device. ${ }^{8,9}$ Recently emerging QCL spectrometers, as used in the present study comprise several (i.e., here four) individually tunable QCLs, thus providing access to a MIR spectral band extending $>1000 \mathrm{~cm}^{-1}$. The utility of QCLs serving as MIR light source in waveguide-based evanescent field absorption sensing scenarios has recently been demonstrated. ${ }^{10-12}$.

Various examples of condensed phase MIR sensing based on ATR crystals or optical fibers have clearly illustrated the advantages of evanescent field absorption spectroscopy. ${ }^{13}$ MIR absorbing analytes present at the waveguide-sample interface interact with the evanescent field resulting in attenuation of the propagating light at analyte-specific absorption frequencies, thus generating an IR-ATR absorbance spectrum. At MIR wavelengths, the evanescent field penetrates only few micrometers into the adjacent analyte matrix, thereby minimizing background absorptions. The absorption of target molecules within the evanescent field establishing a quasi-thinfilm-cell follows a pseudo-Lambert-Beer relationship, which may be described as $\mathrm{A}=(\varepsilon \mathrm{cl}) \mathrm{r}$, where $\varepsilon$ is the molar absorptivity, $\mathrm{c}$ is the concentration of the analyte, 1 is the equivalent optical path length, and $r$ is the fraction of radiation power residing outside the waveguide core (i.e., within the evanescent field). Therefore, enhancing the fraction of energy within the evanescent field emanating from the waveguide surface directly affects the obtainable signal-to-noise ratio (SNR), and thus, the overall attainable sensitivity of the absorption measurement. In turn, the intensity of the evanescent field directly depends on the dielectric constant at the waveguide-sample interface, and in addition, on the dimensions of the waveguide. The latter are largely responsible for the corresponding light mode propagation within the waveguide structure. As the 
dielectric constants are determined by the involved materials, and since the sample properties most frequently may not be freely selected, the most significant sensitivity gain is anticipated from tailoring and optimizing the waveguide structure (i.e., IR-ATR element) itself.

Among the rather limited set of MIR transparent waveguide materials, polycrystalline silver halides ${ }^{14}$ and amorphous chalcogenide glasses ${ }^{15,16}$ are most commonly used. Recently, single mode fibers and planar waveguides manufactured from silver halide have been reported providing transparency up to 20 mm. $^{17}$

A new generation of semiconductor (GaAs/AlGaAs) thinfilm waveguides providing a broad utilizable spectral window from approx. $900 \mathrm{~nm}$ up to $13 \mu \mathrm{m}$ has recently been reported by Mizaikoff and collaborators. ${ }^{10}$ Microfabricating strip waveguides from these materials via photolithography revealed superior sensitivity due to additional lateral mode confinement. ${ }^{18}$ Furthermore, the first on-chip integrated MachZehnder interferometer operating in the MIR was reported providing single mode behavior and exquisite sensitivity. ${ }^{19}$ Alternatively, the utility of mercury-cadmium-telluride (MCT) semiconductor composites ${ }^{20}$, and most recently, thin-film diamond strip waveguides serving as optical transducers for MIR chemical sensing applications was demonstrated. ${ }^{21}$ Furthermore, the integration of single-mode germanium/silicon waveguide structures with a microfluidic architecture was demonstrated for the detection of cocaine in human saliva. ${ }^{22-24}$ However, thin-film MIR waveguide technology usually remains limited to laboratory environments, as delicate waveguide alignment and handling procedures are required.

In the present study, epitaxially grown thin-film GaAs/AlGaAs slab waveguides were modularly packaged into a stainless steel sampling assembly facilitating their usage as highly sensitive transducer for MIR chem/bio sensing applications and spectroscopy. The waveguide mount developed herein provides a cost efficient, robust, and ease-to-handle strategy for manipulating and aligning waveguide structures of microscale dimensions in real-world analytical application scenarios. In particular, it was demonstrated that the optical/analytical performance of thin-film GaAs/AlGaAs slab waveguides in combination with tunable QCLs (tQCLs) and QCL spectrometers promises highly sensitive and miniaturizable MIR analytical platforms, which offers a viable alternative to conventional IR-ATR spectroscopy.

\section{EXPERIMENTAL SECTION}

Based on finite element simulations, thin-film waveguide structures were designed and optimized for two distinct spectral regions. The regime of $1800-1600 \mathrm{~cm}^{-1}$ is of particular interest for protein and aldehyde analysis providing maximum sensitivity via a $6 \mu \mathrm{m} \mathrm{GaAs}$ waveguide structure deposited on top of a $6 \mu \mathrm{m} \mathrm{Al}_{0.2} \mathrm{Ga}_{0.8} \mathrm{As}$ optical buffer layer. The second spectral region of interest extends across $1200-1000 \mathrm{~cm}^{-1}$, and benefits from a $10 \mu \mathrm{m}$ GaAs waveguide layer on top of a $10 \mu \mathrm{m}$ buffer layer. According to these parameters, epitaxially grown GaAs thin-film waveguides based on n-doped GaAs substrates were fabricated by Optowell Co., Ltd (Korea). GaAs wafers were then cleaved along the crystal axis using a diamond knife for obtaining chip dimensions of $5 \times 10 \mathrm{~mm}$. Cleaved chips were placed into a polytetrafluoroethylene (PTFE) inlay, which is in turn mounted into the actual stain- less steel assembly. Finally, the GaAs waveguide was sealed via a PTFE sealing against the stainless steel top plate, as detailed in Figure 1. This design allows the analysis of solid and liquid samples deposited at the waveguide surface along with exchangeability of the GaAs thin-film waveguide and multiple reuse of the mount, e.g., if another waveguide architecture, structure or material is used. The top plate may readily be replaced with a plate comprising a microfluidic infrastructure facilitating continuous flow measurements, e.g., for inline process applications.

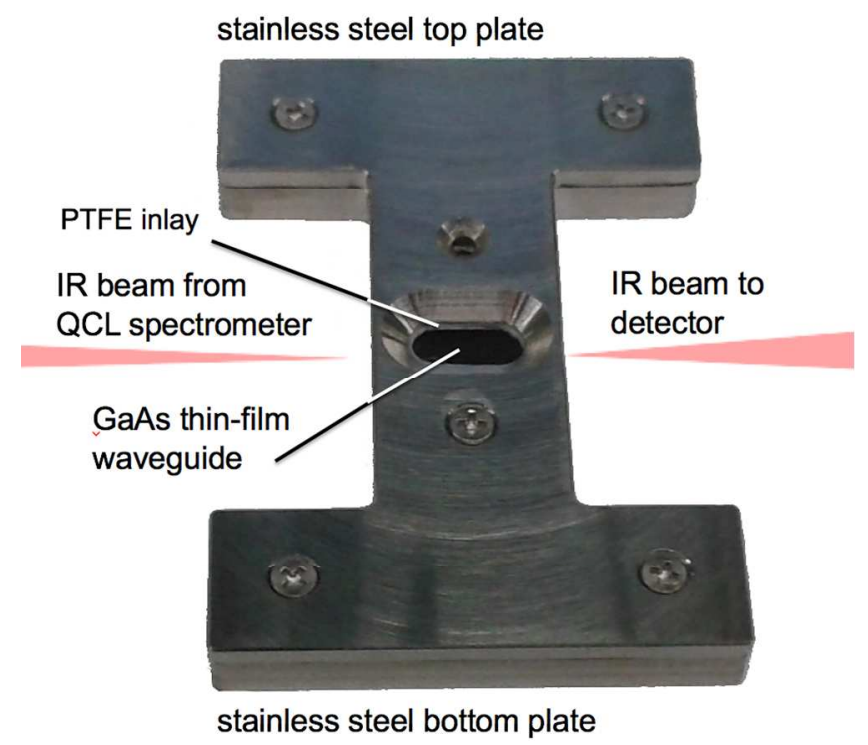

Figure 1. Stainless steel GaAs/AlGaAs thin-film waveguide mounting accessory $(3 \times 5 \mathrm{~cm})$ facilitating evanescent field QCL spectroscopy.

The waveguide mount is designed such that it directly integrates into optical setups using, e.g., commercially available kinematic base plates (here, Thorlabs Inc., Newton, New Jersey, USA) ensuring precise self-realignment within the optical setup after replacement. As the MIR light source, a MIRcat QCL spectrometer (Daylight Solutions Inc., San Diego, CA, USA) comprising four individually tunable QCL modules was used covering a MIR spectral window of $1925-885 \mathrm{~cm}^{-1}$. The QCLs were operated in pulse mode with a pulse repetition rate of $100 \mathrm{kHz}$, and a pulse width of $100 \mathrm{~ns}$. Laser radiation was focused via a $\mathrm{ZnSe}$ lens onto the end facet of the GaAs thinfilm waveguide. A ZnSe lens doublet was then used for focusing radiation emanating at the distal end of the waveguide chip onto a liquid nitrogen cooled MCT detector (Kolmar Technologies, Newbury, MA, USA), which was connected to an oscilloscope and a high-speed digital transient recorder (Saturn, AMO GmbH, Aachen, Germany). Comparative FTIR measurements were performed using a Fourier transform infrared spectrometer (Bruker Alpha, Bruker Optics, Ettlingen, Germany) equipped with an IR-ATR module based on a singlebounce diamond ATR crystal (Platinum ATR, Bruker Optics, Ettlingen, Germany) and a room temperature deuterated Lalanine doped triglycine sulfate (DLaTGS) detector. IR spectra were recorded at a resolution of $4 \mathrm{~cm}^{-1}$ averaging 100 scans per measurement.

Chemicals. Benzaldehyde was purchased from Merck (Darmstadt, Germany) and diethylene glycol monoethyl ether 
(DGME) was obtained from Merck Schuchardt (Hohenbrunn, Germany) both at 'degree for analysis'. $1 \mathrm{M}$ ammonium perchlorate solution was purchased from Sigma-Aldrich. All commercially available chemicals were used as purchased without further purification.

\section{RESULTS AND DISCUSSION}

Chemical sensing using GaAs slab waveguides. For establishing a calibration model for a GaAs slab waveguide-based MIR sensor, a diluted ammonium perchlorate solution was prepared in DI-water. Ammonium perchlorate was used as a model analyte for the wavelength range $1200-1000 \mathrm{~cm}^{-1}$ due to the pronounced absorption feature at $1100 \mathrm{~cm}^{-1}$ (i.e., the stretching vibration of $\mathrm{ClO}_{4}^{-}$). Single wavelength experiments were performed adjusting the QCL to emit at $1100 \mathrm{~cm}^{-1}$ (i.e., $9.1 \mu \mathrm{m})$. The waveguide mount was equipped with a $10 \mu \mathrm{m}$ GaAs waveguide structure, and was aligned such that maximal energy throughput was reached. A volume of $50 \mu \mathrm{L}$ of the analyte solution was applied to the surface of the waveguide structure via an Eppendorf pipet. Accordingly, the intensity of the radiation propagating through the waveguide was dampened via evanescent field absorption following $A=-\log \left(I / I_{0}\right)$. The relationship between absorbance and analyte concentration is shown in Fig. 2. A slope of 0.23 a.u. was derived from a linear fit $\left(\mathrm{R}^{2}=0.998\right)$ describing the obtained sensitivity for a $10 \mu \mathrm{m} \mathrm{GaAs} / \mathrm{AlGaAs}$ slab waveguide. The intensity of the laser pulses was determined via an oscilloscope repeating each measurement 3 times. After each measurement, the GaAs waveguide was cleaned with water, acetone, and isopropyl alcohol. The error associated with these measurements is mainly attributed to minute laser fluctuations. Repeating this study with several GaAs/AlGaAs slab waveguides revealed a reproducible analytical response with less than $5 \%$ variance.

Detection of ammonium perchlorate. The sensor response to $50 \mu \mathrm{L}$ of ammonium perchlorate solutions with concentrations of $0.01,0.05,0.1,0.5$, and $1 \mathrm{M}$ using a $10 \mu \mathrm{m}$, and for comparison a $6 \mu \mathrm{m}$ thick GaAs thin-film waveguide was compared to results recorded via a FTIR spectrometer (Figure 2). The QCL-based measurements reveal comparable results to conventional FTIR-based studies. As expected from computational simulations, the $6 \mu \mathrm{m}$ waveguide provides a higher sensitivity in contrast to the $10 \mu \mathrm{m}$ structure, attributed to the enhanced intensity of the evanescent field with increased mode confinement. However, the overall energy throughput is approximately ten times lower for $6 \mu \mathrm{m}$ waveguides compared to the $10 \mu \mathrm{m}$ structures (i.e the oscilloscope readout of the detector signal was $211 \mathrm{mV}$ for the $6 \mu \mathrm{m}$ waveguides and $2.12 \mathrm{~V}$ for the $10 \mu \mathrm{m}$ waveguide structures), as the coupling efficiency into the waveguide is reduced at smaller waveguide dimensions.

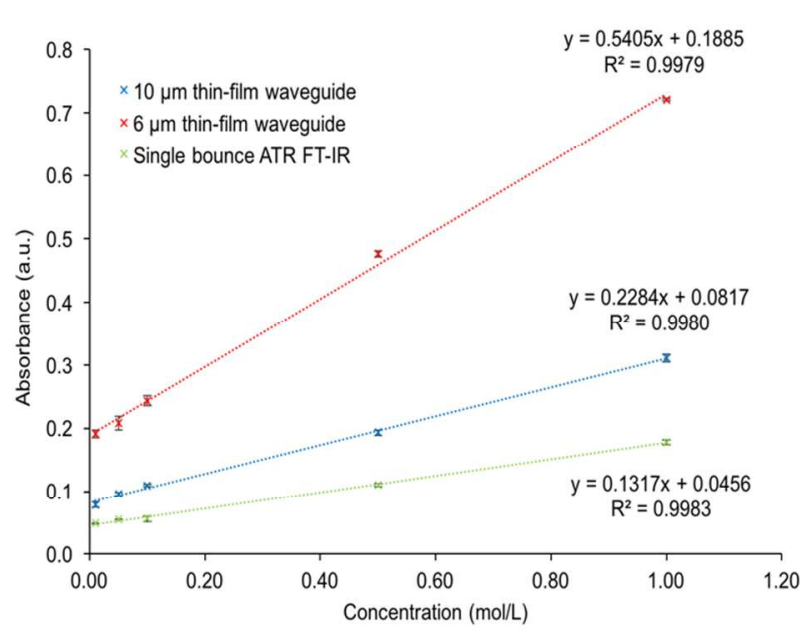

Figure 2. Sensor response to solutions of ammonium perchlorate in water using a $6 \mu \mathrm{m}$ (red) and a $10 \mu \mathrm{m}$ (blue) thick planar GaAs slab waveguide at a wavelength at $1100 \mathrm{~cm}^{-1}$. In comparison, the absorption values recorded with a FTIR spectrometer at $1100 \mathrm{~cm}^{-1}$ are shown (green).

On the basis of DIN 32645, the limit of detection (LOD) for ammonium perchlorate in water was determined at $62.4 \mathrm{mM}$ for the $10 \mu \mathrm{m}$, and at $56.9 \mathrm{mM}$ for the $6 \mu \mathrm{m}$ QCL-coupled waveguide, respectively; using the FTIR spectrometer as the light source yielded $65.3 \mathrm{mM}$ as the LOD. As expected, the sensitivity of the $6 \mu \mathrm{m}$ waveguide is substantial higher compared to the $10 \mu \mathrm{m}$ waveguide. However, the LOD is not significantly improved compared to the $10 \mu \mathrm{m}$ waveguide, as the standard deviation is higher due to the smaller detector signals, which results in larger errors in the signal determination. Nevertheless, the $10 \mu \mathrm{m}$ waveguide still reveals comparable sensitivity and LODs vs. conventional FTIR measurements.

Detection of benzaldehyde. In order to study the overall sensing performance of GaAs waveguides integrated into the macroscopic mount, similar experiments were executed using benzaldehyde solutions in DGME. DGME was used as solvent due to high solubility for benzaldehyde, the low volatility at room temperature, and transparency in the required MIR spectral range. Here, the QCL spectrometer was adjusted emitting at $1700 \mathrm{~cm}^{-1}$ according to the maximum absorbance of the $\mathrm{C}=\mathrm{O}$ stretching band of benzaldehyde. A series of $50 \mu \mathrm{L}$ droplets containing $0.05,0.1,0.25,0.5,0.75$, and $1 \mathrm{M}$ of benzaldehyde solutions in DGME were successively analyzed again using $10 \mu \mathrm{m}$ and $6 \mu \mathrm{m}$ GaAs slab waveguides. After each measurement the GaAs waveguides were cleaned with ethyl acetate, acetone, and isopropyl alcohol. The $6 \mu \mathrm{m}$ waveguide, which was optimized for this wavelength regime revealed the highest sensitivity with a slope of 0.255 a.u., an $\mathrm{R}^{2}$ of 0.999 , and a related LOD of $35.1 \mathrm{mM}$. FTIR studies revealed a slope of 0.0862 , an $\mathrm{R}^{2}$ of 0.998 , and a LOD of $46 \mathrm{mM}$. As expected, the $10 \mu \mathrm{m}$ waveguide offered a reduced sensitivity compared to the $6 \mu \mathrm{m}$ waveguide and to corresponding FTIR studies, as a second mode is propagating within the waveguide resulting in a correspondingly reduced amount of energy within the evanescent field emanating at the surface of the waveguide. 


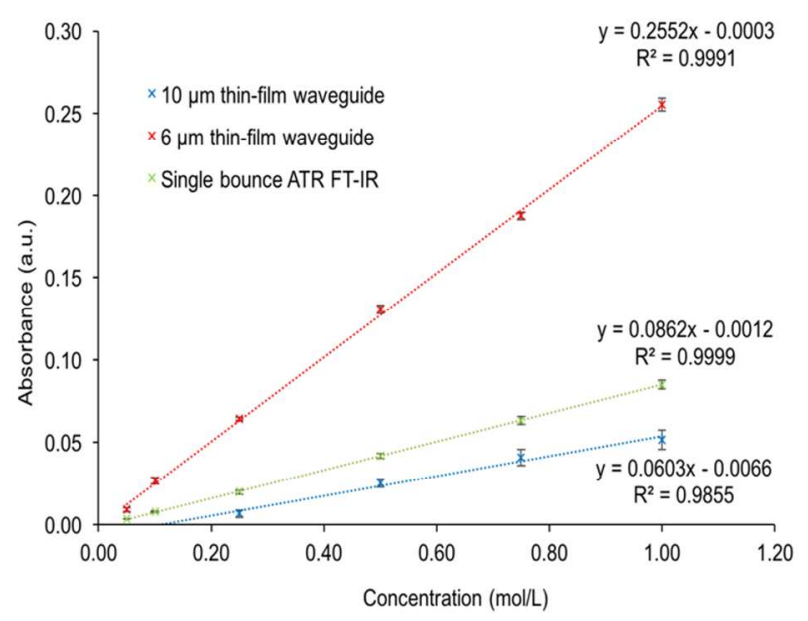

Figure 3. Sensor response to solutions of benzaldehyde in DGME at a $6 \mu \mathrm{m}$ (red) and a $10 \mu \mathrm{m}$ (blue) thick planar GaAs slab waveguide at a wavelength at $1700 \mathrm{~cm}^{-1}$. In comparison, the absorption recorded with a Bruker Alpha at $1700 \mathrm{~cm}^{-1}$ are shown (green).

Broadband chemical detection. In order to demonstrate the utility of broadband QCL-based infrared spectroscopy, ammonium perchlorate solutions were analyzed using a $10 \mu \mathrm{m} \mathrm{GaAs}$ waveguide. The laser emission wavelength was tuned between $1150-1050 \mathrm{~cm}^{-1}$ at a speed of $2 \mathrm{~cm}^{-1} / \mathrm{s}$. Ten thousand Laser pulses were averaged for each data point to obtain a continuous output signal, which was recorded via a high speed digital transient recorder (Saturn, AMO GmbH, Aachen, Germany). Background spectra were recorded against air. QCL-based absorption spectra were calculated following $A=-\log \left(\mathrm{I} / \mathrm{I}_{0}\right)$. Figure 4 exemplarily illustrates single-scan spectra of 0.01 , $0.05,0.1,0.5$, and $1 \mathrm{M}$ ammonium perchlorate solutions deposited onto a $10 \mu \mathrm{m}$ GaAs slab waveguide. For each spectrum, a single background and a single sample scan was performed. The obtained QCL spectra are comparable to single scan spectra recorded with a conventional FTIR spectrometer. However, the difference in absorption between $0.01 \mathrm{M}$ and $0.05 \mathrm{M}$ solutions may still be adequately discriminated within the QCL single scan spectra, while the corresponding single scan FTIR measurements no longer provide distinguishable signals due to the present noise level. While averaging more scans certainly improves the S/N ratio for FTIR studies, significantly longer measurement times are required. Last but not least, while miniaturization of FTIR spectrometers - and accordingly, of interferometers - directly affects the spectroscopic figures-of-merit (e.g., spectral resolution, etc.), miniaturization of (tunable) QCL systems does not detrimentally affect the intensity of the signal or the achievable scan. Hence, QCL-based infrared spectroscopy can provide advantages in analytical scenarios where rapid and sensitive in-situ measurements are demanded in contrast to conventional - usually FTIR-based - spectroscopic techniques.
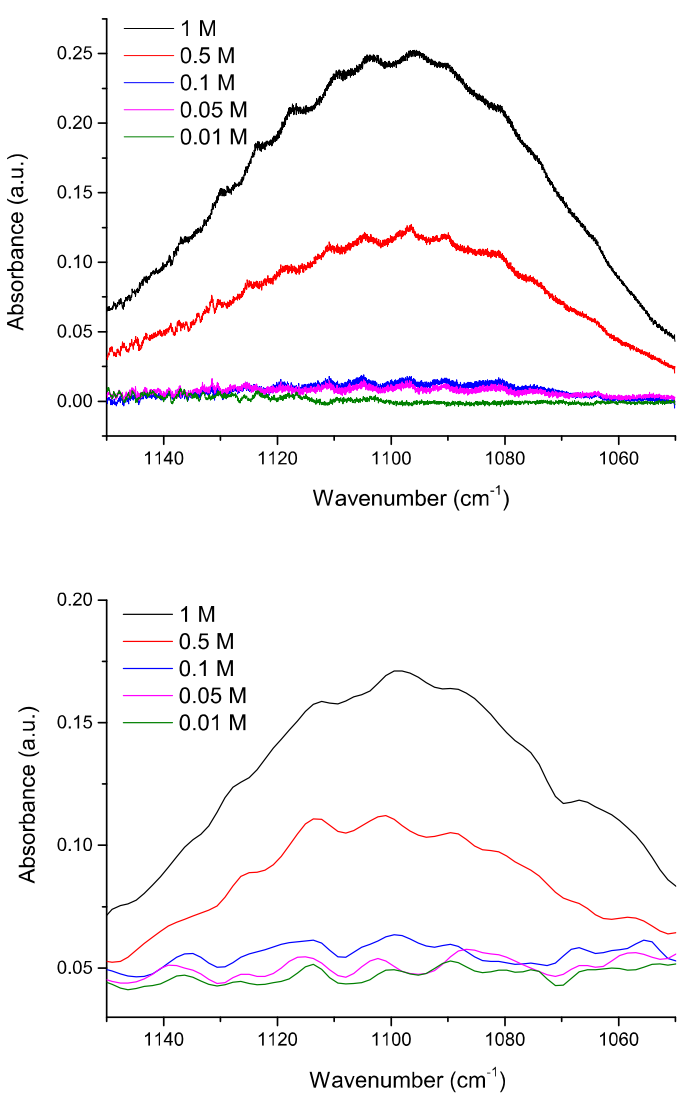

Figure 4. Single scan infrared spectra of ammonium perchlorate solutions in water recorded using the developed waveguide-mount assembly coupled to a QCL spectrometer (top), and recorded with a FTIR spectrometer (bottom).

\section{Conclusions and outlook}

In the present study, a macroscopic accessory for mounting thin-film GaAs/AlGaAs waveguides has been developed, fabricated, and analytically tested. GaAs slab waveguides with dimensions of $5 \times 10 \mathrm{~mm}$ of different thickness $(6$ and $10 \mu \mathrm{m})$ were combined with a tunable QCL spectrometer serving as evanescent field sensing transducers in lieu of conventional ATR crystals. The waveguide mount was designed such that modular adaptation to any IR optical assembly ensuring precise self-realignment is enabled. Moreover, the waveguide chip itself may readily be replaced facilitating that waveguide structures optimized for the desired spectral range may be implemented.

During comparison studies it was confirmed that QCLcoupled thin-film waveguide evanescent field absorption spectroscopy provides comparable sensitivity to conventional FTIR-based measurements, in particular with waveguide dimensions tailored to a specific spectral range of interest. Finally, it was experimentally demonstrated that single-scan QCL spectra provided similar fidelity for the spectroscopic figuresof-merit vs. FTIR spectra obtained by averaging 100 scans. Hence, with the availability of frequency-matched thin-film waveguides packaged into a convenient macroscopic sampling accessory as shown herein, the practical applicability of QCL- 
based IR spectroscopy advances to a yet unprecedented level of utility.

\section{AUTHOR INFORMATIONS}

\section{Corresponding Author}

*E-mail: boris.mizaikoff@uni-ulm.de.

Notes The authors declare no competing financial interest.

\section{ACKNOWLEDGEMENTS}

This study has in part been funded by the European Union's Seventh Framework Programme managed by REA Research Executive Agency http://ec.europa.eu/rea (FP7/2007-2013) under grant agreement no. 314018 FP7-SME-2012-SME (MYCOSPEC). Furthermore, the authors gratefully acknowledge partial support by the Kompetenznetz Funktionelle Nanostrukturen Baden Wuerttemberg, Germany, and by the Center for Integrated Quantum Science and Technology (IQST) @ University of Ulm and University of Stuttgart, Germany. Last but not least, Daylight Solutions, Inc. (San Diego/CA, USA) is thanked for years of fruitful collaboration.

\section{REFERENCES}

(1) Mizaikoff, B. Chem. Soc. Rev., 2013, 42, 8683-8699.

(2) Mizaikoff, B. Anal. Chem. 2003, 75, 258A-267A.

(3) Andanson, J-M.; Baiker, A. Chem. Soc. Rev, 2012, 39, 4571 4584.

(4) Rotermund, F.; Petrov, V.; Noack, F. Opt. Commun. 2000, 185, 177-183.

(5) Kim, S.-S.; Young, C.; Mizaikoff, B. Anal. Bioanal. Chem. 2008, 390, 231-237.

(6) Kazarinov, R. F.; Suris, R. A. Fizika i Tekhnika Poluprovodnikov (Sankt Peterburg) 1971, 5, 797.
(7) Faist, J.; Capasso, F.; Sivco, D. L.; Sirtori, C.; Hutchinson, A. L.; Cho, A. Y. Science 1994, 264, 553.

(8) Saleh, B. E. A.; Teich, M. C. Fundamentals of Photonics, $2^{\text {nd }}$ ed.; John Wiley \& Sons, New Jersey, 2007, pp. 644-673.

(9) Capasso, F.; Gmachl, C.; Sivco, D. L.; Cho, A. Y. Phys. Today 2002, 55, 34.

(10) Charlton, C.; Giovannini, M.; Faist, J.; Mizaikoff, B. Anal. Chem. 2006, 78(12), 4224-7.

(11) Young, C.; Kim, S.-S.; Luzinova, Y.; Weida, M.; Arnone, D.; Takeuchi, E.; Day, T.; Mizaikoff, B. Sens. Act. B. 2009, 140, 24.

(12) Charlton, C.; Katzir, A.; Mizaikoff, B. Anal. Chem. 2005, 77, 4398.

(13) Janotta, M.; Vogt, F.; Voraberger, H.; Waldhauser, W.; Lackner, J. M.; Stotter, C.; Beutl, M.; Mizaikoff B, Anal. Chem. 2004, 76,384 .

(14) Taga, K.; Kellner, R.; Kainz, U.; Sleytr, U. B. Anal. Chem. $1994,66,35-39$.

(15) Karlowatz, M.; Kraft, M.; Mizaikoff, B. Anal. Chem. 2004 76, 2643-2648.

(16) Grille, R.; Martin, G.; Labadie, L.; Arezki, B.; Kern, P.; Lewi, T.; Tsun, A.; Katzir, A., Opt. Express 2010, 17, 12516-12522.

(17) Houizot, P.; Boussard-Plédel, C.; Faber, A. J.; Cheng, L. K.; Bureau, B.; Van Nijnatten, P. A.; Gielesen, W. L. M.; Pereira do Carmo, J.; Lucas, J. Opt. Express 2007, 15, 12529-12538.

(18) Wang, X.; Kim, S.-S.; Roßbach, R.; Jetter, M.; Michler, P.; Mizaikoff, B. Analyst 2012, 137, 2322-2327.

(19) Sieger, M.; Balluff, F.; Wang, X.; Kim, S.-S; Leidner L.; Gauglitz, G.; Mizaikoff, B. Anal. Chem. 2013, 85, 3050-3052.

(20) Wang, X.; Antoszewski, J.; Putrino, G.; Lei, W.; Faraone, L.; Mizaikoff, B. Anal. Chem. 2013, 85 (22), 10648-10652

(21) Wang, X.; Karlsson, M.; Forsberg, P.; Sieger, M.; Nikolajeff, F.; Österlund, L.; Mizaikoff, B. Anal. Chem. 2014, 86, 8136-8141

(22) Chang, Y.-C.; Paeder, V.; Hvozdara, L.; Hartmann, J.-M.; Herzig, H. P. Opt. Lett. 2012, 37, 2883-2885.

(23) Chang, Y.-C.; Wägli, P.; Paeder, V.; Homsy, A.; Hvozdara, L.; Wal, P.; Francesco, J. D.; Rooij, N. F.; Herzig, H. P. Lab chip 2012, 12, 3020-3023.

(24) Wägli, P.; Chang, Y.; Homsy, A.; Hvozdara, L.; Herzig, H. P.; Rooij, N. F. Anal. Chem. 2013, 85, 7558-7565.

\section{TOC}

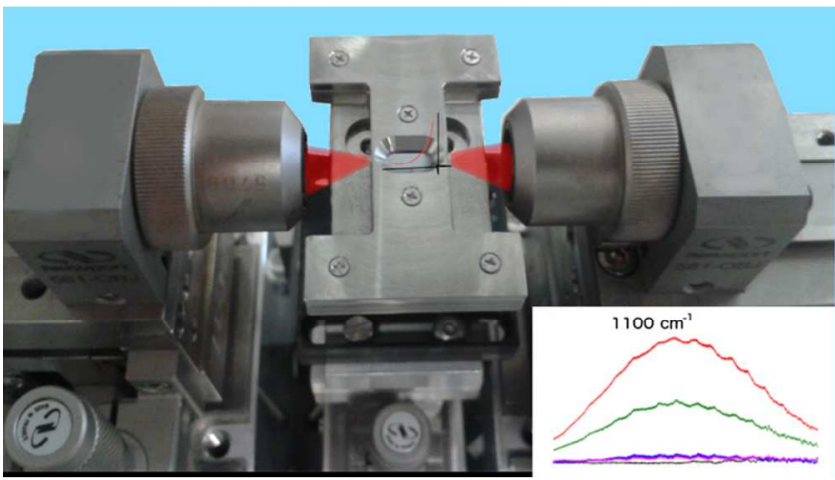

